P-IWerte um 20\% nach oben, dies basierend auf Experimente, in denen der Inhibitoreffekt verschieden großer Mengen Kieselgel bestimmt und, ausgedrückt als Farbverminderung, zu 11\% - 27\% gefunden wurde.

Die aufgrund der statistischen Berechnungen gefundene Genauigkeit der Methode darf für die Werte der Gesamtlipoide, der Phosphatide, des Lecithins und Sphingomyelins als befriedigend bezeichnet werden. Beim Lysolecithin mit einem Variationskoeffizienten von $\pm 10 \%$ wäre eine größete Genauigkeit sehr wünschenswert, hauptsächlich im Hinblick auf seine eventuelle Wichtig- keit bei der Komplement-Lyse. Sehr fragwürdig bleibt wie bereits erwähnt die Bestimmung des Kephalins (Variationskoeffizient $\pm 34 \%$ ). Die Fehlerbreite bei der von uns vorgeschlagenen Methode ist allerdings immer noch wesentlich geringer als die Variabilität der Lipoidzusammensetzung bei normalen Sera. Tatsächlich scheint heute das. Problem der Feststellung signifikanter $\mathrm{Ab}$ weichungen vom Norm-Wert weit eher durch statistisch einwandfreie Festlegung der Normalverteilung der Gesamtlipoide, als durch die Genauigkeit der analytischen Prozeduren bestimmt zu sein.

\title{
Literatur
}

1. Kaufmann, H. P., Fette und Seifen 46, 268 (1939). - 2. Wren, J. J., J. Chromatogr. 4, 173-195 (1960). - 3. Marinetrr, G. V., J. Lipid Res. 3, 1 (1962). - 4. Wercker, H., Klin. Wochenschr. Jg. 37, Heft 14, 763 (1959). - 5. Mangold, H. K., Fette, Seifen und Anstrichmittel 61, 877 (1959). - 6. WAGNER, H., L. HöRHAMMER und P. WolfF, Biochem. Zeitschr. 334, 175-184 (1961). - 7. Blank, M. L., J. A. Schmit und O. S. Privett, J. Am. Oilchemists Soc. 41, Nr. 5, 371 (1964). - 8. Christian, J. C., S. Jakovic und D. Yr-Yung Hsia, J. Lab. Clin. Med. 5,5 (1964). - 9. Doizakr, W. M. und L. Zieve, Proc. Exp. Biol. Med. 113, 91 (1963). - 10. HabermanN, E. G., G. Bandflow und B. KRusche, Klin. Wschr. 39, 816 (1961). - 11. JatzKewITz, H., a) Hoppe Seyler's Zeitschr. physiol. Chemie 326, 61 (1961), b) Hoppe Seyler's Zeitschr. physiol. Chemie 336, 25 (1964). 12. Robinson, N. und B. M. Phillips, Clin. Chim. Acta 8, 385 (1963). - 13. Skipski, V. P., R. F. Peterson, J. Sanders und M. Barclay, J. Lipid Res. 4, No. 2, 227 (1963). - 14. Vrkrot, O.,
Acta Med. Scand. 175, Fasc. 4, 443 (1964). - 15. VoGEL, W. C. W. M. DoizAkI und L. ZIEVE, J. Lipid Res. 3, 138 (1962). 16. Zöllner, N. und G. Wolfram, Klin. Wochenschr. Jg. 40, Heft 21, 1101 (1962). - 17. Frsk, C. H. und Y. Subba-Row, J. Biol. Chem. 66, 375 (1925). - 18. De Iongh, van PeLt, J. G., J. Lipid Res. 3, No. 3, 385 (1962). - 19. Folch, J., M. LeEs und G. H. Sloane Stanley, J. Biol. Chem. 226, 497 (1957). 20. Bloor, W. R., J. Biol. Chem. 77, 53 (1928). - 21. RENKonen, O., T. U. Kosunen und O. V. Renkonen, Ann. Med. Exp. Biol. Fannia 41, 375 (1963). - 22. Bligh, E. G. und W. J. Dyer, Can. J. Biochem. Physiol. 37, 8 (1959). - 23. Jestrng, E. und H. O. BANG, Scand. J. Clin. Lab. Invest. 15, 654 (1963). - 24. BARTLETT, G. R., J. Biol. Chem. 234, 466 (1959). - 25. Shrn, Y. S., Anal. Chem. 34, 1164 (1962). - 26. Hanson, S. W. F. und J. Olley, Biochem. J. 89, 101 p (1963). - 27. Privetr, O. S., M. L. Blank, D. W. Codding und E. C. Nrckell, J. Am. Oil Chemist Soc. 42, Nr. 5, 381 (1965).

\section{Untersuchungen zur enzymatischen Bestimmung der Galaktose mit Galaktoseoxydase}

\author{
Von H. Förster und M. HASLbeck
}

Aus dem Institut für vegetative Pbysiologie der Universität Frankfurt (Direktor: Prof. Dr. med. E. Heinz) und der III. Medizinischen Klinik des Krankenbauses München-Schivabing (Chefarzt: Priv. Doz. Dr. med. H. Mebnert)

(Eingegangen am 3. Januar 1967)

Die quantitative Galaktosebestimmung mittels Galaktoseoxydase-Peroxydase wurde untersucht. Dabei wurde als optimales Puffersystem Barbitalnatrium-Natriumacetat-Puffer gefunden. - Als Wasserstoffdonator wirkte o-Tolidin am günstigsten. Bestimmungen im Serum und besonders im Harn werden durch Harnsäure gestört. Dabei dienen Harnsäure und auch Glutathion als Wasserstoffdonatoren für die Peroxydasereaktion. Diese beiden Substanzen lassen sich jedoch auf einfache Weise durch Zusatz von Quecksilberacetat zu der als Enteiweißungsmittel dienenden Perchlorsäure eliminieren. Ein weiterer Vorteil des Quecksilberacetatzusatzes ist die dadurch bewirkte Farbvertiefung des Chromogens.

The quantitative determination of galactose by means of the coupled galactose-oxidase peroxidase system was investigated. Sodium barbiturate-sodium acetate buffer was found to be the most suitable system for this work. O-tolidine was the most satisfactory hydrogendonor. Measurements in serum and particularly in urine were disturbed by the presence of uric acid. This is because uric acid and also glutathione may act as hydrogen donors for the peroxidase reaction. These two substances may be simply eliminated by the addition of mercuric acetate to the perchloric acid used as a protein precipitant. A further advantage of mercuric acetate is that it intensifies the colour of the chromogen.

Die Bestimmung der Galaktose im Blut war bisher immer schwierig, da eine spezifische Methode fehlte. Die im Blut vorhandene Glucose stört bei jedem chemischen Nachweis, der auf dem Reduktionsprinzip oder auf dem Furfurolprinzip beruht (1); denn die Blutglucosekonzentration ist meist wesentlich höher als die Blutgalaktosekonzentration, während die chemischen Nachweismethoden für beide Zucker etwa gleich empfindlich sind. Die Reduktionsmethoden exfassen zudem noch andere reduzierende Substanzen als sog. Restreduktion, wäh- rend die o-Toluidin-Methode relativ spezifisch für Hexosen ist. Eine gewisse Verbesserung brachte die von Watson (2) angegebene Methode. Glucose wird durch Glucoseoxydase zerstört und die von diesem Ferment nicht angegriffene Galaktose wird anschließend mit oToluidin bestimmt. $\mathrm{Da}$ aber die Glucoseoxydation mit Glucoseoxydase meist unvollständig ist, sind die Fehlermöglichkeiten dieser Methode immer noch groß (3). Neue Möglichkeiten ergaben sich aus der Isolierung der Galaktoseoxydase aus Dactylium dendroides (4-8). Die 
Bestimmung der Galaktose mit Galaktoseoxydase erfolgt analog der Glucosebestimmung mit Glucoseoxydase. Es entsteht jedoch nicht (entsprechend der Gluconsäure) Galaktonsäure, sondern D-Galaktohexodialdose. Mehrere Autoren haben sich bereits mit der Galaktosebestimmung mittels Galaktoseoxydase-Peroxydase beschäftigt (4-10). $\mathrm{Da}$ wir für unsere Fragestellungen einen einfachen und spezifischen Galaktosenachweis benötigten, wurden von uns Untersuchungen mit dem von der Fa. Worthington in den Handel gebrachten gereinigten Enzym Galaktoseoxydase sowie mit Testpackungen "Galactostat" der gleichen Firma durchgeführt.

\section{Methodik}

Galaktoseoxydase wurde von der Fa. Worthington bezogen, Peroxydase von der $\mathrm{Fa}$. Boehringer.

Als U'asserstoffdonatoren wurden o-Dianisidin ( $\mathrm{Fa}$. Merck), o-Dianisidinhydrochlorid (Fa. Boehringer), o-Tolidin (Fa. Merck), oToluidin ( $\mathrm{Fa}$. Merck), Resorcin (Fa. Mcrck) und o-Kresol (Fa. Merck) auf ihre Eignung untersucht.

Neben dem von der Fa. Worthington empfohlenen Phosphatpuffer $(0,15 \mathrm{M})$ wurden folgende weitere Puffer geprüft: Trispuffer $(0,15 \mathrm{M})$, Malcinsäure-Trispuffer $(0,2 \mathrm{M})$, Citronensäure-Phosphatpuffer $(0,3 \mathrm{M})$ Äthanolaminpuffer $(0,15 \mathrm{M}), \beta, \beta$-Dimethylglutarsäurepuffer $(0,1 \mathrm{M})$ und Barbitalnatrium-Acctatpuffer $(0,15 \mathrm{M})$.

Zur Enteineißung wurden 3,6-proz. Perchlorsäure, 0,16-proz. Uranylacetat, 5-proz. Trichloressigsäure, sowie eine Lösung 1:1 aus $0,025 \mathrm{M}$ Quecksilberacetat in 1-proz. Essigsäure und 6-proz. Perchlorsäurc verwendet.

D^neben wurde die Wirkung von Harnstoff, Harnsäure, Kreatinin, Glutathion und Ascorbinsäure auf die Farbentwicklung geprüft.

Die Einstellung der Puffer erfolgte mit einem elektrischen pHMeter (Glaselektrode). Die Extinktion wurde mit einern Photometer "Eppendorf" bestimmt; o-Dianisidin und o-Tolidin wurden bei Filter $436 \mathrm{~m} \mu$, o-Toluidin bei Filter $366 \mathrm{~m} \mu$ und o-Kresol bei $405 \mathrm{~m} \mu$ abgelesen.

Testansätze: Galaktoseoxydase und Peroxydase wurden in $100 \mathrm{ml}$ des entsprechenden Puffers gelöst. Von den Chromogenen wurden 1-proz. Lösungen teils in aqua dest., teils in Methanol hergestellt. Für die Untersuchungen wurden Verdünnungsreihen von 1 bis $150 \mathrm{mg} \%$ Galaktose angesetzt. Zur Enteiweißung wurde $0,1 \mathrm{ml}$ Probe mit $1,0 \mathrm{~m} l$ Enteiweißungsflüssigkeit versetzt.

Anfänglich wurde mit folgendem Testansatz in Anlehnung an die Testvorschrift der $\mathrm{Fa}$. Worthington gearbeitet: $0,2 \mathrm{ml}(0,5 \mathrm{ml})$ Probe wurde mit $2,0 \mathrm{ml}(4,0 \mathrm{ml})$ Puffer-Enzym-Chromogenlösung bei $37^{\circ}$ inkubiert. Nach $60 \mathrm{Min}$. wurden $3,0 \mathrm{ml}(5,0 \mathrm{ml}) 0,25 \mathrm{M}$ Glycinpuffer ( $\mathrm{pH} 9,7$ ) zugesetzt.

Für weitere Bestimmungen erwies sich der folgende Ansatz als besonders geeignet: $0,1 \mathrm{~m} l$ enteiweißte Probe mit $3,0 \mathrm{~m} l$ PufferEnzym-Chromogenlösung werden $60 \mathrm{Min}$. bei $37^{\circ}$ inkubiert. Glycinpuffer wurde nicht zugesetzt. Als am besten geeignetes Puffersystem erwies sich $0,15 \mathrm{M}$ Barbitalnatrium/0,15 M Natriumacetat (Michaelispuffer (8)), auf $\mathrm{pH} 8,1$ eingestellt.

o-Tolidin muß vor Zugabe von Barbitalnatriumpuffer vollständig in Wasser oder Methanol $(0,5-1 \mathrm{ml})$ gelöst sein; andernfalls tritt Kristallbildung ein.

\section{Ergebnisse}

Mit dem von der Fa. Worthington für die „Galactostat"Testpackung vorgeschlagenen Testansatz lassen sich nur für den Bereich von 12,5-50 mg\% Galaktose brauchbare Ergebnisse erzielen. Der Zusatz von Glycinpuffer führt durch Alkalisierung zu einer Hemmung der Enzymreaktion und damit zur Stabilisierung der Farbe. Glycin reagiert mit dem als Chromogen verwendeten o-

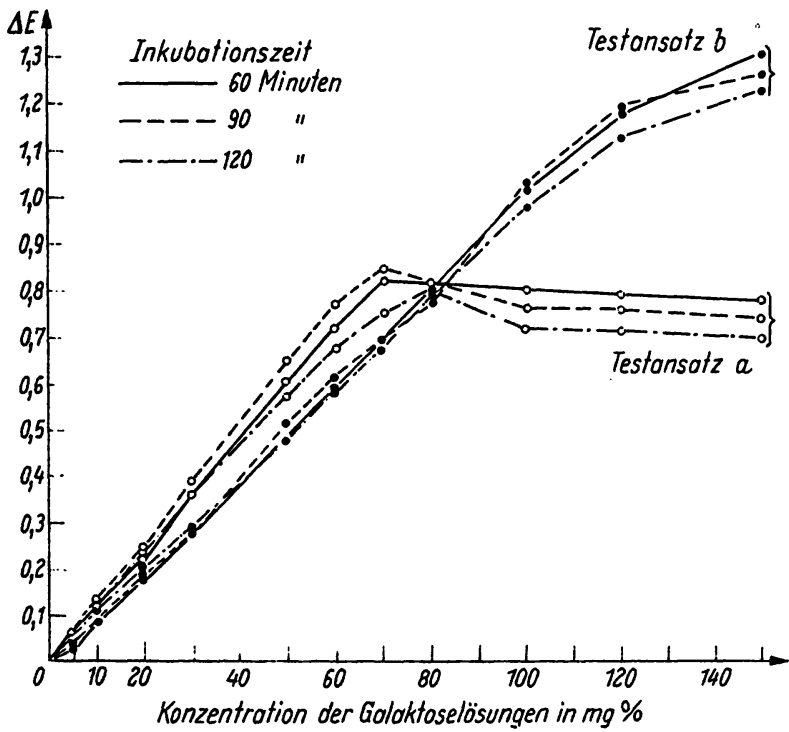

Abb. 1

Einfluß von Inkubationszeit und Glycinpufferzusatz auf die enzymatische Bestimmung der Galaktose mit Galaktoseoxydase (Barbitalnatrium - Natriumacetatpuffer $0,15 \mathrm{M}$ )

Testansatz a: $0,2 \mathrm{ml}$ Probc und $2,0 \mathrm{ml}$ Puffer-Enzymlösung (80 E Galaktoseoxydase, $10 \mathrm{mg}$ Peroxydase, $15 \mathrm{mg}$ o-Tolidin pro $100 \mathrm{ml}$ ). Am Ende der Inkubation Zusatz von $3,0 \mathrm{~m} /$ Glycinpuffer $(0,25 \mathrm{M}, \mathrm{pH} 9,7)$.

Testansatz $b: 0,2 \mathrm{~m} l$ Probe und $5,0 \mathrm{~m} /$ Puffer-Enzymlösung (33,3 E Galaktoseoxydase, $4 \mathrm{mg}$ Peroxydase, $6 \mathrm{mg}$ o-Tolidin pro $100 \mathrm{~m} l)$

Tolidin, so daß die Extinktionen $0,6-0,8$ nicht überschreiten (Abb. 1). Zudem sind die Eichkurven mit Phosphatpuffer teilweise nicht linear und schwer reproduzierbar.

Wie aus Abbildung 2 ersichtlich ist, erwiesen sich $\beta, \beta$ Dimethylglutarsäurepuffer, Barbitalnatrium-Natriumacetatpuffer, Phosphatpuffer und Trispuffer als brauchbar. Nach Enteiweißung mit Perchlorsäure blieb bei Verwendung von Trispuffer die Farbreaktion aus. Mit Phosphatpuffer angesetzte Lösungen waren nicht einmal

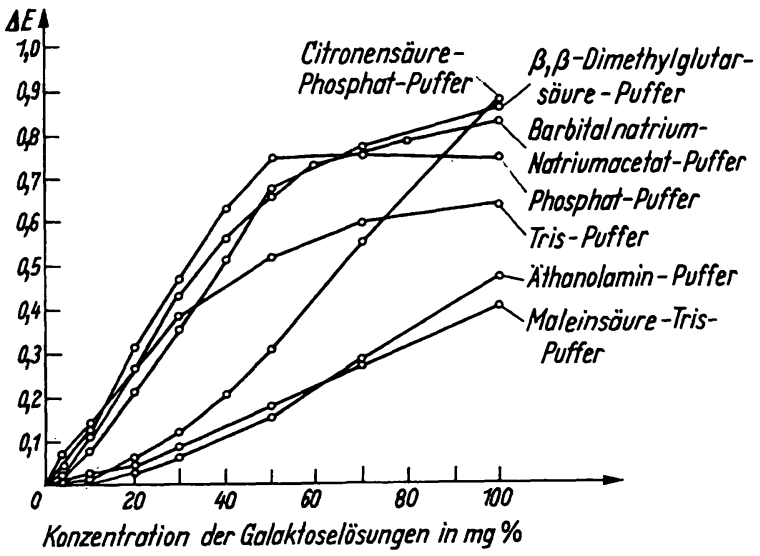

Abb. 2

Einfluß verschiedener Puffersysteme auf die Farbentwicklung bei der enzymatischen Galaktosebestimmung

Testansatz: $0,5 \mathrm{~m} /$ Probe und $4,0 \mathrm{ml}$ Puffer-Enzymlösung (40 E Galaktoseoxydase, $5 \mathrm{mg}$ Peroxydase, $10 \mathrm{mg}$ o-Tolidin pro $100 \mathrm{ml}$ ). Am Ende der Inkubation (60 Min., $37^{\circ}$ ) Zusatz von $5,0 \mathrm{ml}$ Glycinpuffer $(0,25 \mathrm{M}, \mathrm{pH} 9,7)$ 
im Kühlschrank 48 Stunden haltbar. Außerdem war die Eichkurve bei Perchlorsäureenteiweißung nur bis zu einerExtinktion von 0,5 linear. Die höheren Extinktionen wichen reproduzierbar ab. Demgegenüber halten sich mit dem Michaelispuffer angesetzte Lösungen länger als eine Woche, und die Eichkurven sind über fast den doppelten Bereich linear. Aus Abbildung 2 ist außerdem ebenfalls die Wirkung des Glycinzusatzes zu erkennen. Verschiedene Autoren $(4-7,10,11)$ hatten bereits oKresol und o-Dianisidin, sowie o-Tolidin als Chromogene vorgeschlagen. o-Dianisidin, das z. B. für die Glucoseoxydase-Peroxydasereaktion als Wasserstoffdonator allgemein verwendet wird, scheint die Galaktoseoxydase kompetitiv zu hemmen (6) und ist deshalb nicht brauchbar (Abb. 3). o-Kresol, von Fischer und ZAPF
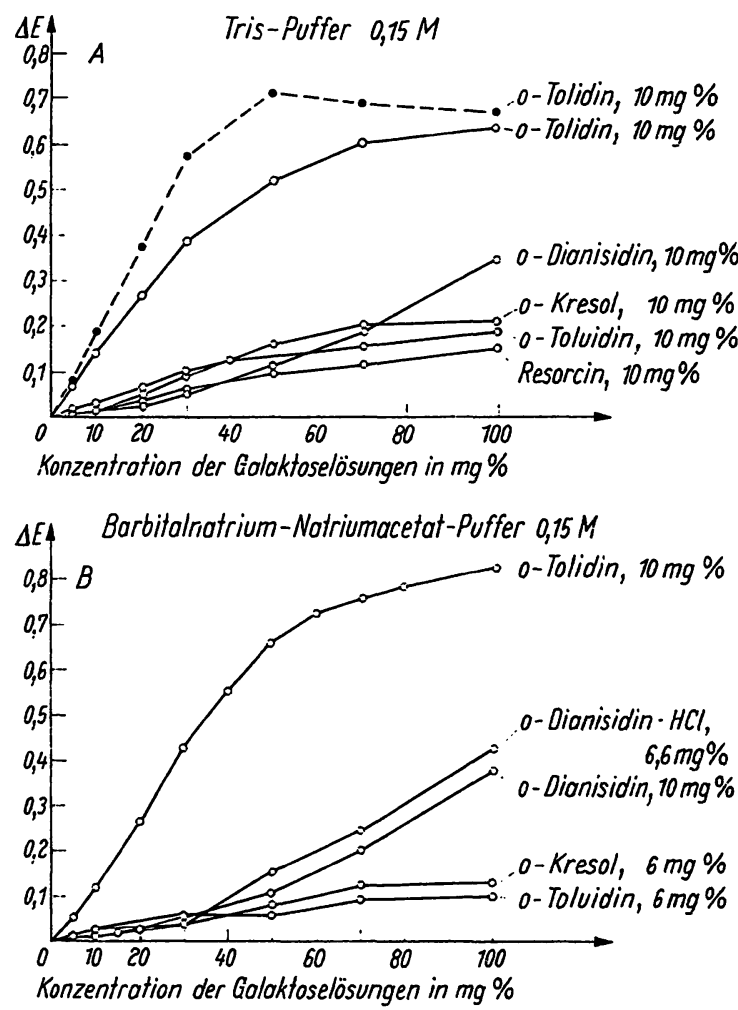

Abb. 3

Einfluß verschiedener Chromogene auf die Farbentwicklung beim Nachweis der Galaktose mit Galaktoseoxydase

Testansatz: $0,5 \mathrm{~m} /$ Probe und $4,0 \mathrm{ml}$ Puffer-Enzymlösung (40 E Galaktoseoxydase bzw. $80 \mathrm{E}$ bei durchbrochener Linie, $5 \mathrm{mg}$ Peroxydase, pro $100 \mathrm{ml}$ ). Am Ende der Inkubation (60 Min., 37 ) Zusatz von $5,0 \mathrm{~m} l$ Glycinpuffer $(0,25 \mathrm{M}, \mathrm{pH} 9,7)$

(6) vorgeschlagen, sowie o-Toluidin und Resorcin sind als Wasserstoffdonatoren ungeeignet. Lediglich o-Tolidin, bereits früher von WATSON (12) für die Glucoseoxydase-Peroxydasemethode verwendet, führt zu brauchbaren Ergebnissen (Abb. 3). Die Abweichungen von der Linearität in dieser Abbildung bei höheren Extinktionen sind auf den Glycinzusatz zurückzuführen.

Bei unseren Untersuchungen lag das pH-Optimum für die Galaktoseoxydase-Peroxydasereaktion etwa bei $\mathrm{pH}$ 7,4 in Phosphatpuffer und in Glycinpuffer, und bei $\mathrm{pH} 8,1$ in Michaelispuffer (Abb. 4). Die Verschiebung des $\mathrm{pH}$ Optimums von Enzymreaktionen in verschiedenen

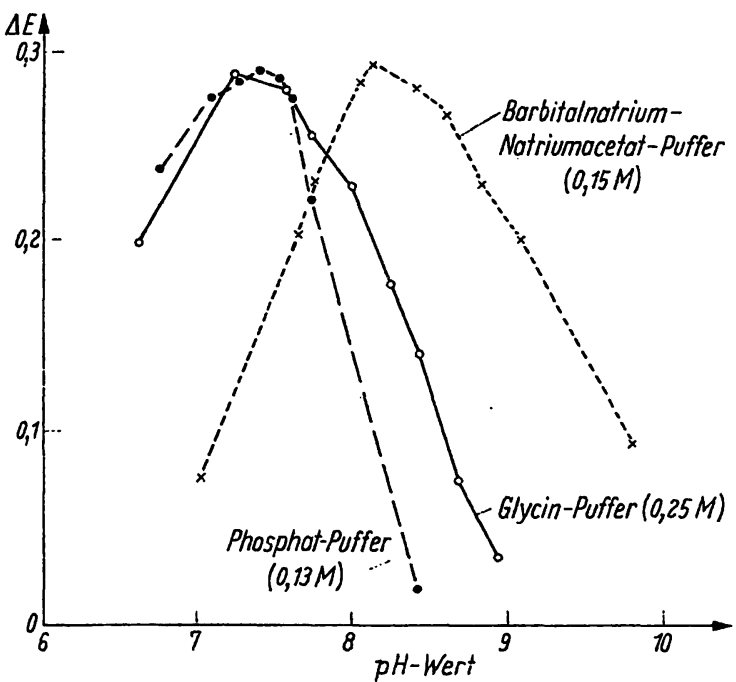

Abb. 4

Die Abhängigkeit der Farbentwicklung vom $\mathrm{pH}$-Wert bei der enzymatischen Galaktosebestimmung

Testansatz: $0,1 \mathrm{~m} l$ Probe und $3,0 \mathrm{ml}$ Puffer-Enzymlösung

(30 E Galaktoseoxydase, $4 \mathrm{mg}$ Peroxydase, $6 \mathrm{mg}$ o-Tolidin pro $100 \mathrm{ml}$ ). Inkubation: $60 \mathrm{Min}$. bei $37^{\circ}$

Puffersystemen wurde bereits mehrfach beschrieben (13). Glycinpuffer ist für diesen Ansatz ungeeignet, da der basische $\mathrm{p} K$ von Glycin bei $\mathrm{pH} 9,8$, also weit außerhalb des $\mathrm{pH}$-Optimums liegt.

$\mathrm{Da}$ die Ergebnisse mit der „Galactostat"“-Testpackung nicht immer gleichmäßig waren, wurde versucht, durch Modifikationen günstigere Ergebnisse zu erzielen. Als günstigste Kombination fanden wir: $30 \mathrm{E}$ Galaktoseoxydase, $4 \mathrm{mg}$ Peroxydase und $6 \mathrm{mg}$ o-Tolidin pro $100 \mathrm{ml}$ 0,15M Michaelispuffer ( $\mathrm{pH} 8,1$ ). Ohne Zusatz von o-Tolidin ist die Enzymlösung mindestens eine Woche haltbar. Nach Zusatz des Chromogens sollte sie rasch verbraucht werden.

Eine Hemmung der Glucoseoxydase-Peroxydasereaktion

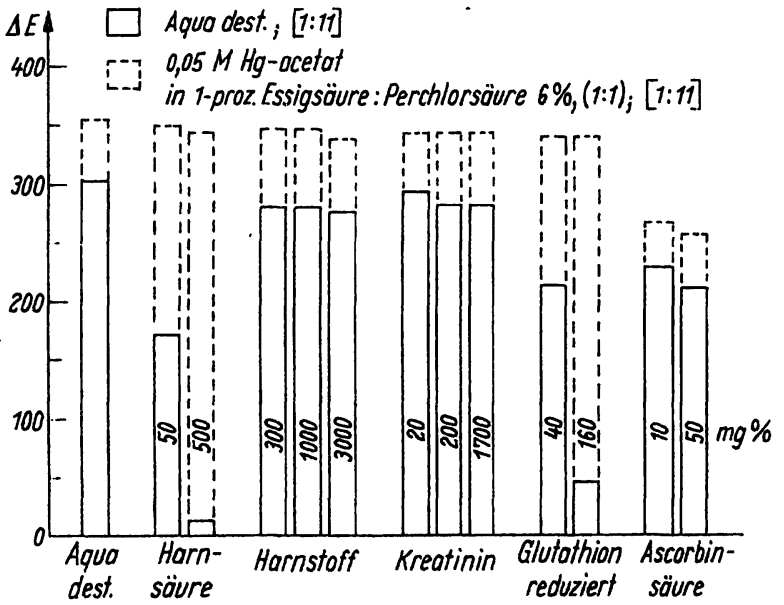

Abb. 5

Einfluß von Hemmsubstanzen bei der enzymatischen Bestimmung derGalaktose mit Galaktoseoxydase

Testansatz nach Enteiweißung $1: 11: 0,1 \mathrm{~m} /$ Probe und $3,0 \mathrm{~m} /$ PufferEnzymlösung

(30 E Galaktoseoxydase, $4 \mathrm{mg}$ Peroxydase, $6 \mathrm{mg}$ o-Tolidin pro $100 \mathrm{~m} /$ ); Inkubation: $60 \mathrm{Min}$. bei $37^{\circ}$ 
durch Glutathion, Cystein und Ascorbinsäure ist beschrieben worden $(14,15,16)$. Fischer und $Z_{\text {App }}(6,11)$ machten auf das ähnliche Verhalten der Galaktoseoxydase-Peroxydasereaktion aufmerksam. Außerdem haben diese Autoren eine Beeinflußung der Reaktion durch Harnstoff beschrieben. Beim Nachweis der Glucose im Blut sind wegen der hohen Konzentration die Abweichungen, die durch diese störenden Substanzen verursacht werden, unerheblich. $\mathrm{Da}$ die $\mathrm{Ga}$ laktosekonzentration in den Proben meist viel niedriger liegt, können deutliche Abweichungen bei dem Nachweis mit Galaktoseoxydase-Peroxydase auftreten. Aus Abbildung 5 geht hervor, daß nur Harnsäure, reduziertes Glutathion und Ascorbinsäure die Galaktoseoxydase-Peroxydasereaktion wesentlich stören; Harnstoff und Kreatinin stören sehr wenig. Durch Zusatz von Quecksilberacetat zur Enteiweißungslösung werden Harnsäure und Glutathion offensichtlich vollständig ausgefällt (Abb. 5). Von den stärker störenden Substanzen bleibt lediglich Ascorbinsäure in Lösung. Von anderen geprüften Schwermetallionen störten Silberionen die Enzymreaktion; durch Uranyl wurde die Harnsäure nicht ausgefällt (Abb. 6). Entfernung von Ascorbinsäure aus dem Untersuchungsmaterial ist meist nicht erforderlich, da Ascorbinsäure im biologischen Untersuchungsgut kaum je in höheren Konzentrationen auftritt.

Die Harnsäure- und Glutathionwirkung wird darauf zurückgeführt, daß diese Substanzen als Wasserstoffdonatoren für die Peroxydasereaktion wirken können (6, 11). In Abbildung 7 ist zu erkennen, daß die Farbentwicklung bei der Galaktoseoxydase-Peroxydasereaktion durch Harnsäure proportional zur zugesetzten Menge gehemmt wird. Jedoch wird auch die höchste zugesetzte Harnsäuremenge quantitativ durch Quecksilberacetat ausgefällt (Abb. 7). Ein weiterer Vorteil des

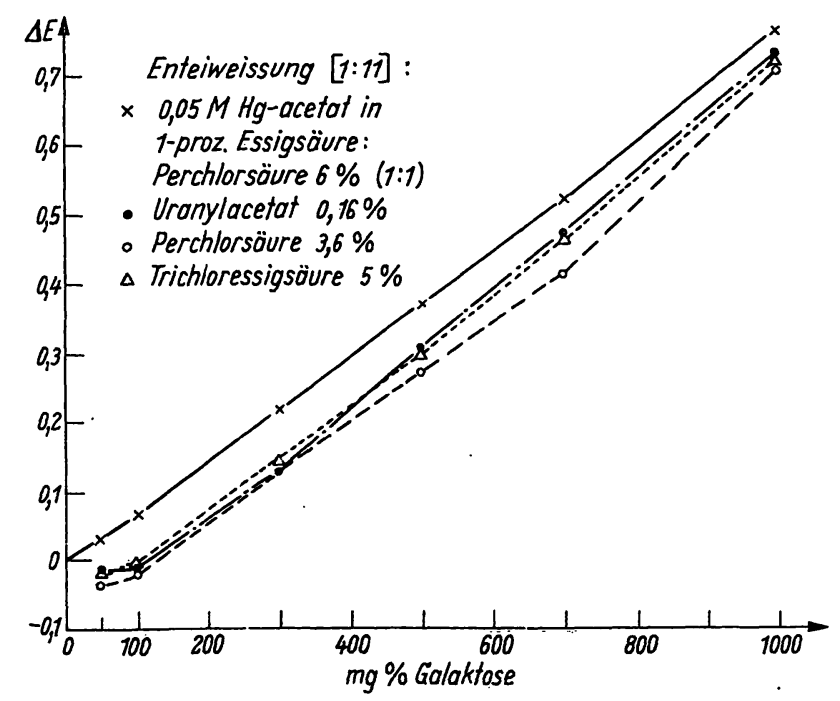

Abb. 6

Einfluß von Harnsäure auf die enzymatische Bestimmung der Galaktose mit Galaktoseoxydase

Testansatz nach Enteiweißung $1: 11: 0,1 \mathrm{~m} /$ Probe und 3,0 $\mathrm{m} l$ PufferEnzymlösung

(30 E Galaktoseoxydase, $4 \mathrm{mg}$ Peroxydase, $6 \mathrm{mg}$ o-Tolidin pro $100 \mathrm{~m} /$ ). Inkubation: $60 \mathrm{Min}$. bei $37^{\circ}$

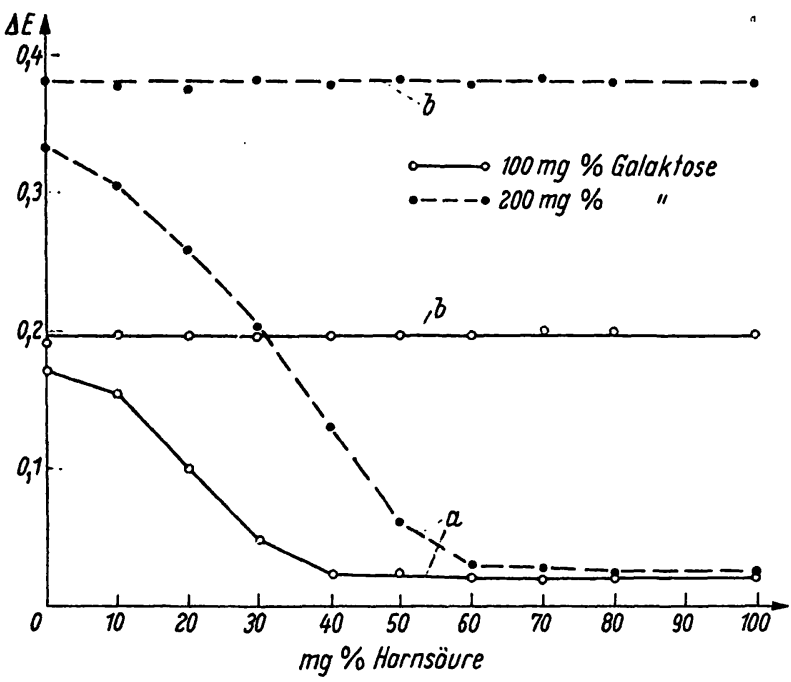

Abb. 7

Einfluß steigender Harnsäurekonzentrationen bei der enzymatischen Bestimmung der Galaktose mit Galaktoseoxydase

Testansatz nacb Enteineißung 1:11 mit Percblorsäure-Quecksilberacetat: $0,1 \mathrm{~m} /$ Probe und $3,0 \mathrm{~m} /$ Puffer-Enzymlösung

(30 E Galaktoseoxydase, $4 \mathrm{mg}$ Peroxydase, $6 \mathrm{mg}$ o-Tolidin pro $100 \mathrm{~m} /$ ). Inkubation: $60 \mathrm{Min}$. bei $37^{\circ}$

Quecksilberacetatzusatzes ist die durch diesen Zusatz bewirkte reproduzierbare Extinktionszunahme um etwa $10-15 \%$ (Abb. 5 u. 7). Bei Galaktosebestimmungen im Harn wird die störende Harnsäure durch Versetzen der Harnprobe mit $0,05 \mathrm{M}$ Quecksilberacetatlösung vollständig eliminiert. Ein unerwarteter Vorteil des Quecksilberacetatzusatzes zur Perchlorsäure bei der Enteiweißung besteht in einer reproduzierbaren Farbvertiefung von o-Tolidin. Die Extinktion der Eichkurve bei Perchlorsäureenteiweißung mit Quecksilberacetatzusatz war um etwa $15 \%$ höher als nach Verwendung von Perchlorsäure ohne Zusatz (Abb. 5 u. 7).

\section{Diskussion}

Bisher beschäftigten sich mehrere Autoren mit der aus Polyporus circinatus isolierten Galaktoseoxydase (4-8, 10, 11). Im Gegensatz zur Glucoseoxydase scheint die Galaktoseoxydase kein Flavoprotein zu sein; die prothetische Gruppe ist noch unbekannt $(4,5)$. Das Reaktionsprodukt ist D-Galaktohexodialdose, im Gegensatz zu Gluçonsäure bei der Glucoseoxydasereaktion. Der Pyranosering ist essentiell, da Galaktonsäure kaum angegriffen wird, während 1,5-Anhydrogalaktit mit der gleichen Geschwindigkeit wie Galaktose oxydiert wird. Auch Galaktoside werden oxydiert, wobei kein Anhaltspunkt dafür besteht, daß vorher eine Hydrolyse eintritt $(4,5)$. Andere Galaktosederivate werden wenig angegriffen.

Fast alle bisherigen Untersuchungen wurden mit Phosphatpuffer durchgeführt $(4-7,10)$. Lediglich Rorr und Mitarbeiter (8) verwendeten Glycinpuffer bei $\mathrm{pH} 8,4$. $\mathrm{Da}$ der basische $\mathrm{p} K$ von Glycin bei $\mathrm{pH} 9,8$ liegt, ist bei $\mathrm{pH} 8,4$ nur eine geringe Pufferwirkung zu erwarten (Abb. 4). Wegen verschiedener oben beschriebener 
Nachteile haben wir bei unseren Untersuchungen Barbitalnatrium-Acetatpuffer bei $\mathrm{pH}$ 8,1 verwendet und damit gut reproduzierbare Ergebnisse erzielt.

Als Wasserstoffdonator für die Peroxydasereaktion scheint das bereits früher von WATSON (12) verwendete o-Tolidin am besten geeignet (Abb. 2). o-Dianisidin hemmt die Galaktoseoxydasereaktion kompetitiv, wie Fischer und ZAPF $(6,11)$ nachgewiesen haben (Abb. 2). Allerdings fanden andere Autoren bei Verwendung von o-Dianisidin lineare Eichkurven $(7,10)$. Bei Verwendung von o-Kresol $(6,11)$ sind die Eichkurven zu flach, um genaue Bestimmungen zu ermöglichen (Abb. 2).

Die störende Wirkung verschiedener Faktoren im Blut war bereits früher aufgefallen. VERDIER und HJELM (10) stellten Eichkurven im Serum der untersuchten Personen auf, um die störende Wirkung der verschiedenen Faktoren zu eliminieren. Rотн und Mitarbeiter (8) haben keine näheren Angaben über die Art der hemmenden Substanzen im Blut gemacht. Enteiweißung wurde mit Bariumhydroxyd-Zinksulfat durchgeführt. Diese Enteiweißung ist jedoch sehr umständlich und wird durch die erforderliche genaue Einstellung der Reagenzienlösung kompliziert. Fischer und $\operatorname{ZAPF}_{\text {APF }}(6,11)$ haben darauf hingewiesen, daß die Harnsäure und reduziertes Glutathion wahrscheinlich als Wasserstoffdonatoren für die Peroxydasereaktion dienen können. Auch Harnstoff und Kreatinin wird von diesen Autoren eine deutliche Hemmwirkung zugeschrieben. In dem von uns verwendeten Testansatz störten jedoch lediglich Harnsäure, Glutathion und sehr hohe Konzentrationen von Ascorbinsäure. Frscher und ZApr (11) eliminierten die störenden Substanzen durch Inkubation der Proben mit Ionenaustauschern. Auch dieses Verfahren ist für Routineuntersuchungen zu umständlich. Ein wesentlicher Vorteil der von uns verwendeten Enteiweißung mit Quecksilberacetat-Perchlorsäure liegt daher in der einfachen Durchführbarkeit. Durch Quecksilberacetat werden die Enzymreaktionen nicht beeinflußt, die störenden Substanzen mit Ausnahme von Ascorbinsäure jedoch vollständig eliminiert.

Bei Vorgehen nach der Testvorschrift der Fa. Worthington sind gegenüber unserer Modifikation zwei zusätzliche Pipettierungen erforderlich. Daneben wurde der Harnsäure- und Glutathioneinfluß nicht berücksichtigt. Auch gegenüber den von anderen Autoren angegebenen Nachweisvorschriften $(6,7,8,10)$ zeichnet sich die von uns durchgeführte Modifikation besonders durch den geringeren Zeitaufwand aus; daneben wurden alle störenden Faktoren ausgeschaltet.

\title{
Literatur
}

1. Förster, H. und H. Mehnert, Methoden der Kohlenhydratbestimmungen. Hdb. Kinderheilkunde Bd. II/1, S. 754. SpringerVerlag, Bcrlin-Heidelberg-New York (1966). - 2. Watson, D., Analyt. Chem. 5, 260 (1963). - 3. FörSTER, H., Unveröffentl. Ergebn. - 4. Avigad, D., D. Amaral, C. Asenio und B. L. HorECKER, J. biol. Chemistry 237, 2736 (1962). - 5. CoOper, J. A. D., W. Smith, M. Bacita und M. Medina, J. biol. Chemistry 234, 445 (1959). - 6. FISCHER, W. und J. ZAPF, Hoppe-Seylers Z. physiol. Chem. 337, 186 (1964). - 7. Ford, J. D. und J. C. Haworth, Clin. Chem. (New York) 11, 1002 (1964). - 8. Roth, H., S. SEgaL und D. Bertor, Analyt. Biochem. (New York) 10, 32 (1965). -
9. MrChaelis, L., Biochem. Z. 234, 139 (1931). - 10. Verdier, C. H. DE und M. HJELM, Clin. chim. Acta (Amsterdam) 7, 742 (1962). - 11. FrsCHER, W. und J. ZAPF, Hoppe-Seylers Z. physiol. Chem. 339, 54 (1964). - 12. WAtson, D., Analyt. Biochem. (New York) 3, 131 (1962). - 13. Axelrod, J., J. Pharmacol. exp. Therapeut. 138, 28 (1962). - 14. Kutrer, D., Aerztl. Laborat. 7, 175 (1961). - 15. Scrmidr, F. H., Methoden der Blut- und Harnzuckerbestimmung unter besonderer Berücksichtigung enzymatischer Verfahren, S. 76. Fortschr. Diabetesforsch., Georg Thieme Verlag, Stuttgart (1963). - 16. Schultherss, F. R., Helvet. med. acta 33, 59 (1966).

Dr. H. Förster

6 Frankfurt/Main, Ludwig-Rehn-Str. 14

\section{Bestimmung der Oxydation von Östradiol zu Östron im menschlichen Organismus mit Hilfe von Östradiol-17 $\alpha-T$}

\author{
Von M. WenzeL und K.-U. BLuM \\ Auls dem Pbysiologisch-chemischen Institut (Direktor: Prof. Dr. Dr. E. Scbütte) und der I. Medizinischen Klinik \\ (Direktor: Prof. Dr. Dr. l. c. H. Frh. v. Kress) der Freien Universität Berlin
}

(Eingegangen am 20. Februar 1967)

Es wird eine Methode beschrieben, mit der das Mindestausmaß der biologischen Oxydation von Oestradiol zu Oestron im Gesamtorganismus gemessen werden kann. Von dem injizierten Oestradiol-17 $\alpha-\mathrm{T}$ wird durch die Oestradioldehydrogenase das Tritium auf Pyridinnucleotide übertragen und letztlich zu Tritiumwasser verbrannt. Die Zunahme der Konzentration des Tritiumwassers im Gesamtkörperwasser gilt als Maßstab für die Oxydation des Steroids. Die Zeitabhängigkeit der Oestradioloxydation bei einigen Versuchspersonen wird angegeben. Bei Patienten ohne pathologischen Leberbefund werden innerhalb von 24 Stdn. mindestens $50-70 \%$ des injizierten Oestradiols oxydiert und nur 3\% im Urin als $17 \alpha$-T-Steroid ausgeschieden.

A method is described for measuring the oxidation of oestradiol to oestrone in the whole organism. The tritium of injected oestradiol-17 $\alpha-\mathrm{T}$ is transferred to pyridine nucleotides by oestradiol dehydrogenase and finally metabolised to tritiated water. The increase in the concentration of tritiated water in the total body water is a measure of the oxidation of the steroid. The time course of oestradiol oxidation is given for some experimental persons. In patients with no pathological liver condition at least $50-70 \%$ of the injected oestradiol is oxidised within $24 \mathrm{hrs}$. and only $3 \%$ is excreted in the urine as $17 \alpha-\mathrm{T}$ steroid. 